\title{
Implementação do Nível F do MR-MPS com Práticas Ágeis do Scrum em uma Fábrica de Software
}

\author{
Edmar Catunda $^{1}$, Camila Nascimento ${ }^{1}$, Cristina Cerdeiral ${ }^{2}$, Gleison Santos $^{3}$, \\ Elaine Nunes ${ }^{2}$, Natália Chaves Lessa Schots ${ }^{2}$, Marcelo Schots ${ }^{2}$, Ana Regina Rocha ${ }^{2}$ \\ ${ }^{1}$ Rightway Consultoria \& Sistemas - Rua do Rosário 103, $12^{\circ}$ andar - Centro \\ CEP 20041-004 - Rio de Janeiro, Brasil \\ ${ }^{2}$ COPPE/UFRJ - Universidade Federal do Rio de Janeiro \\ Caixa Postal 68511 - CEP 21945-970 - Rio de Janeiro, Brasil \\ ${ }^{3}$ Programa de Pós-Graduação em Informática - Universidade Federal do Estado do Rio \\ de Janeiro (UNIRIO) - Caixa Postal 56011- CEP 22290-970 - Rio de Janeiro, Brasil

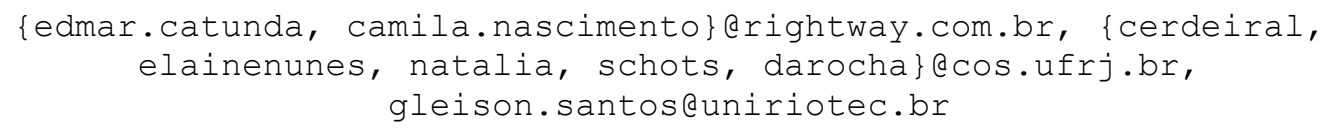

\begin{abstract}
This paper describes the methodology used in the process improvement initiative of Rightway Consultoria \& Sistemas towards MR-MPS level $F$ with the support of Scrum's agile practices. This paper presents the standard development process phases containing Scrum's agile practices and the challenges and lessons learned in the implementation of each process expected for MPS.BR in an agile environment.
\end{abstract}

Resumo. Este artigo descreve a metodologia utilizada na iniciativa de melhoria de processos de software da Rightway Consultoria \& Sistemas objetivando alcançar o nível F do MR-MPS em conjunto com práticas ágeis da metodologia ágil Scrum. Este artigo apresenta ainda as fases do processo padrão de desenvolvimento contendo as práticas da metodologia Scrum e os desafios e as lições aprendidas na implementação de cada processo previsto pelo MPS.BR em um ambiente ágil.

\section{Introdução}

A Rightway Consultoria \& Sistemas é uma empresa com sede no Rio de Janeiro, especializada em desenvolvimento de soluções e projetos de Tecnologia da Informação que atua em quatro linhas de serviços: sistemas sob medida, infraestrutura, business intelligence (BI) e alocação de pessoal. O principal objetivo da empresa que a levou a investir na implementação do nível F do MR-MPS [Softex 2009b] foi assegurar aos clientes a prestação de serviços com alto nível de qualidade. Em 2009, quando começou a iniciativa de melhoria de processos, a empresa já utilizava algumas práticas ágeis da metodologia Scrum [ScrumAlliance 2011] na execução de seus projetos.

Dessa forma, a empresa desejava implementar processos para organizar melhor o desenvolvimento de software, permitindo um maior controle e alcançando uma melhor qualidade nos seus produtos, sem perder, no entanto, a agilidade nos seus projetos. A empresa foi avaliada com sucesso no nível F do MR-MPS [Softex 2009b] em dezembro de 2010. Este artigo descreve a metodologia utilizada para implantar as 
melhorias de processos necessárias para alcançar o nível $\mathrm{F}$ do modelo (Seção 2), as fases do processo padrão de desenvolvimento com as práticas da metodologia Scrum [ScrumAlliance 2011] adotadas e as adaptações necessárias (Seção 3), os desafios e particularidades da implementação de cada processo em um ambiente ágil (Seção 4) e as lições aprendidas com esta implementação (Seção 5). Os autores esperam que estas informações possam auxiliar outras empresas e consultorias na combinação de práticas ágeis e modelos de maturidade e capacidade em seus processos de desenvolvimento.

\section{Metodologia de Implementação}

A Rightway Consultoria \& Sistemas, juntamente com a consultoria da COPPE/UFRJ, iniciou a implementação das melhorias de processo necessárias para alcançar o nível $\mathrm{F}$ do MR-MPS [Softex 2009b], seguindo a estratégia SPI-KM [Santos et al. 2007a], na qual a gerência de conhecimento é utilizada para auxiliar a implementação de melhorias de processo de software. As fases da estratégia utilizada podem ser observadas na Figura 1 e são descritas a seguir.

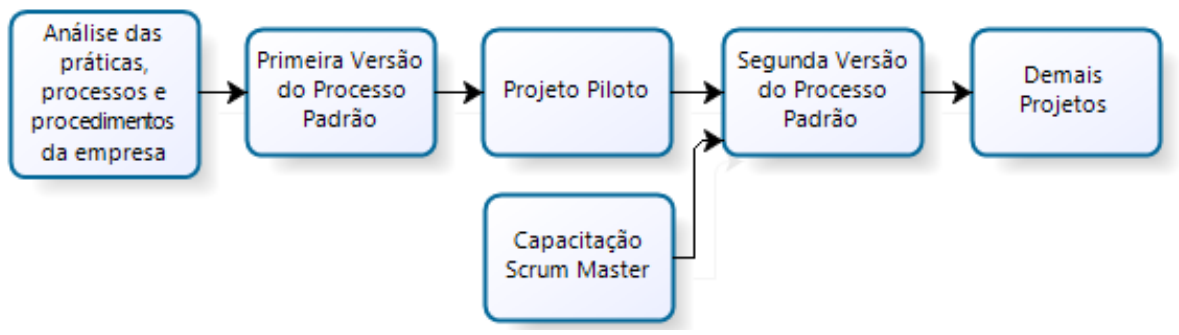

Figura 1 - Fases da Estratégia de Implementação

i. Análise das práticas, processos e procedimentos da empresa: As práticas, os processos e procedimentos já utilizados pela empresa foram analisados, com o objetivo de identificar e entender as necessidades da empresa, permitindo a elaboração de um processo padrão que atendesse a estas necessidades. Neste momento, a empresa colocou a necessidade de manter a agilidade de seus processos adequada ao tamanho e duração dos projetos (cerca de dois a três meses normalmente) e ao ciclo de vida utilizado.

ii. Primeira Versão do Processo Padrão: Assim, a primeira versão do processo padrão de desenvolvimento foi elaborada buscando manter as práticas ágeis já utilizadas (descritas ao longo deste artigo) e atender às necessidades identificadas na fase anterior. O objetivo desta primeira versão é permitir que a empresa utilize o processo rapidamente em um projeto piloto e aprenda com esta experiência, evoluindo o processo e adequando-o à realidade da empresa.

iii. Projeto Piloto: O processo padrão foi instanciado e utilizado em um primeiro projeto com o apoio das ferramentas do ambiente TABA [Santos et al. 2007b]. Por meio desta execução do processo, a empresa identificou várias oportunidades de melhoria no processo padrão, dentre elas a substituição do ambiente TABA por ferramentas mais dinâmicas para apoiar o uso do processo pelos projetos [Catunda et al. 2010].

iv. Capacitação Scrum Master: Em paralelo à execução do projeto piloto, a gerência da Fábrica e todos os coordenadores de projetos (que agem como gerentes dos projetos) fizeram o curso e se tornaram Certified Scrum Masters, aumen- 
tando o conhecimento da empresa nesta metodologia ágil.

v. Segunda Versão do Processo Padrão: As oportunidades de melhoria identificadas na execução do projeto piloto e a capacitação Certified Scrum Masters geraram novas sugestões de alteração no processo padrão. Foi elaborada, então, uma segunda versão do processo padrão, mais voltada para as práticas do Scrum e mais adaptada à realidade da empresa.

vi. Demais Projetos: O processo padrão revisto foi utilizado nos demais projetos da empresa com o apoio de ferramentas em tarefas importantes previstas por seus processos, tornando mais ágil sua execução [Catunda et al. 2010].

\section{Processo e Práticas do Scrum}

A segunda versão do processo padrão de desenvolvimento da empresa possui cinco fases, que podem ser observadas na Figura 2. As três primeiras fases são obrigatórias, pois ocorrem em todos os projetos da empresa; as duas últimas fases são opcionais, pois nem sempre se encontram no escopo dos projetos.

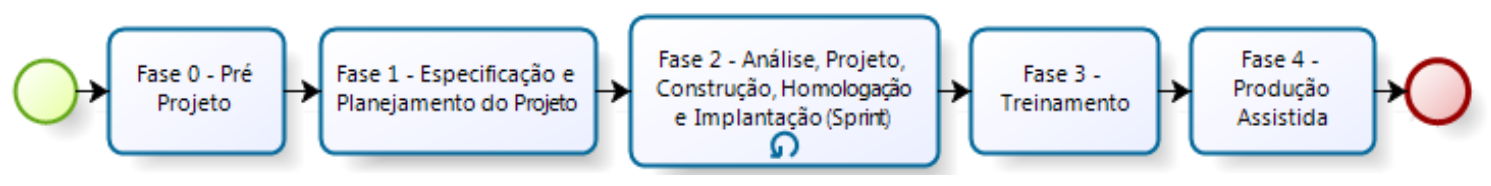

Figura 2 - Processo Padrão de Desenvolvimento

\subsection{Fase 0 - Pré-Projeto}

A Fase 0 contempla as atividades que são executadas antes de o projeto iniciar, para a elaboração da Proposta Técnica sempre que uma nova oportunidade é identificada. Nesta fase, são realizadas as atividades do processo Gerência de Portfólio de Projetos, o entendimento inicial dos requisitos, as estimativas iniciais para o projeto (utilizando um método próprio da empresa, baseado na complexidade das funcionalidades e nas tecnologias utilizadas) e a análise inicial do projeto. As práticas da metodologia Scrum não são utilizadas neste momento.

\subsection{Fase 1 - Especificação e Planejamento do Projeto}

A Fase 1 contempla o planejamento e a especificação técnica do projeto, e pode ser observada na Figura 3. Inicialmente, é realizado um planejamento para a Fase 1, seguido do levantamento e análise dos requisitos, englobando a identificação dos requisitos de cliente (já parcialmente identificados na Fase 0), dos requisitos funcionais e não funcionais e dos casos de uso. Com base no levantamento e análise dos requisitos, as estimativas são refinadas e é realizado um planejamento macro para todo o projeto.

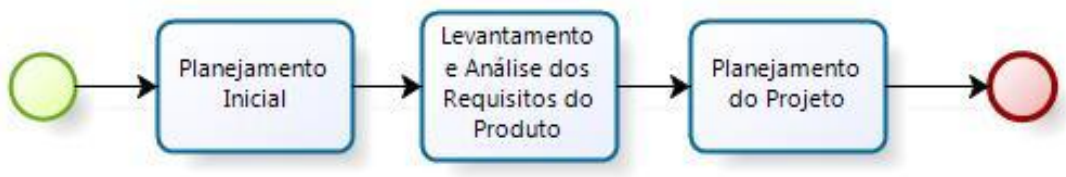

Figura 3 - Fase 1 do Processo Padrão de Desenvolvimento

As práticas da metodologia Scrum utilizadas nesta fase são a divisão do projeto em sprints de duração fixa (usualmente, duas semanas) e o Release Planning, no qual 
são realizadas a estimativa do número de sprints necessários e uma alocação prévia dos casos de uso nos sprints, gerando uma estimativa inicial de finalização do projeto. As adaptações realizadas pela empresa nas práticas da metodologia Scrum são: (i) a utilização de casos de uso no lugar das estórias, mantendo a compatibilidade com o método de estimativas criado pela empresa, e (ii) a utilização de um método de estimativa próprio de complexidade por caso de uso e tecnologias utilizadas no projeto com o apoio da base histórica no lugar da velocidade da equipe.

\subsection{Fase 2 - Análise, Projeto, Construção, Homologação e Implantação (Sprint)}

A Fase 2 representa um sprint e tem a duração fixa de uma ou duas semanas, sendo esta última a mais usual. Nesta fase, são realizadas as atividades relacionadas com a análise, projeto, construção, homologação e implantação de alguns casos de uso do projeto, como pode ser observado na Figura 4. Esta fase pode ser iterativa, caso mais de um sprint seja definido para o projeto.

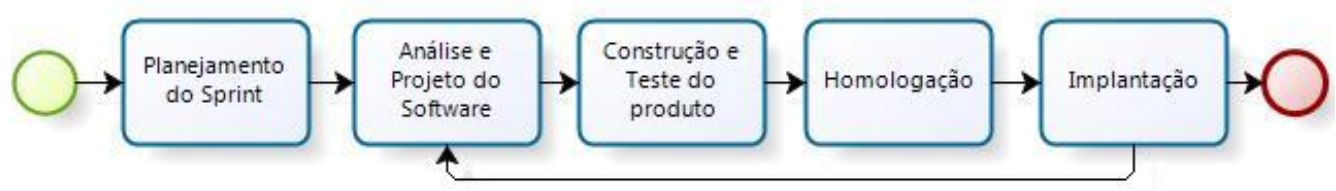

Figura 4 - Fase 2 do Processo Padrão de Desenvolvimento

O sprint é iniciado com o seu planejamento, no qual são utilizadas as práticas da metodologia Scrum Sprint Planning 1 (na qual os casos de uso a serem desenvolvidos no sprint atual são selecionados) e Sprint Planning 2 (na qual os casos de uso selecionados para o sprint são detalhados e analisados), sempre que possível com a participação do cliente ou com seu representante. Cada caso de uso é, então, trabalhado por toda a equipe do projeto, desde a sua análise até sua homologação interna, na ordem de maior valor para o cliente. Somente após a equipe concluir o primeiro caso de uso do sprint, o segundo caso de uso começa a ser trabalhado. Desta forma, a fase 2 é uma iteração no ciclo de vida do projeto, e dentro dela ocorrem iterações de desenvolvimento para cada caso de uso. Um sprint possui normalmente cerca de 1 a 3 casos de uso.

Durante todo o sprint, são utilizadas as seguintes práticas da metodologia Scrum: Dashboard (quadro contendo a situação dos casos de uso do Sprint), Burndown Chart (gráfico contendo o andamento do desenvolvimento dos casos de uso) e as reuniões Daily Sprint para monitoração do projeto, com a participação do coordenador do projeto e da equipe do projeto. São realizadas reuniões semanais do coordenador do projeto com a gerência da Fábrica nas quais o andamento do projeto naquela semana é apresentado, juntamente com os impedimentos ainda não solucionados, e são definidas ações para tratá-los. O sprint se encerra com as reuniões Sprint Review, na qual as funcionalidades desenvolvidas são apresentadas para o cliente, homologadas e implantadas, e Sprint Retrospective, na qual são coletadas lições aprendidas.

Em alguns projetos, o cliente não apresenta interesse em receber versões intermediárias do produto e solicita receber apenas uma versão no final do desenvolvimento. Neste caso, as atividades de homologação e implantação são removidas dos sprints intermediários e são mantidas apenas no último sprint do projeto. As reuniões Sprint Review e Sprint Retrospective, no caso de o cliente (ou seu representante) não poder participar, ocorrem apenas com a participação da equipe do projeto, do coordenador do 
projeto e da gerência da Fábrica.

\subsection{Fase 3 - Treinamento}

Esta fase contempla o planejamento e a execução dos treinamentos necessários para o uso e operação do produto pelo cliente, e não é obrigatória por não fazer parte do escopo de todos os projetos da empresa. Nesta fase não são utilizadas práticas da metodologia Scrum.

\subsection{Fase 4 - Produção Assistida}

Esta fase contempla o planejamento e a execução do período de produção assistida, no qual a empresa tira dúvidas sobre a utilização do produto e corrige problemas encontrados pelo cliente. Esta fase não é obrigatória por não se encontrar no escopo de todos os projetos da empresa. Caso sejam identificados problemas a serem corrigidos, novos sprints são planejados para o projeto, repetindo as atividades descritas na Fase 2.

\section{Desafios Encontrados na Implementação}

Por se tratar de um ambiente ágil com práticas da metodologia Scrum, alguns desafios e particularidades foram encontrados na implementação das melhorias de processo necessárias para atingir o nível F do modelo MR-MPS [Softex 2009b]. Os desafios relacionados à implementação de cada processo pertencente ao nível $\mathrm{F}$ do modelo são discutidos nas seções seguintes, com a exceção de Aquisição, declarado fora de escopo.

\subsection{Gerência de Projetos}

O principal desafio com relação a este processo foi manter a agilidade dos projetos. Com este objetivo, foram estudadas algumas ferramentas de apoio voltadas para projetos ágeis e a empresa resolveu adotar o Redmine [Redmine 2011]. De forma geral, o Redmine é utilizado para o planejamento e controle das atividades do projeto [Catunda et al. 2010]. O planejamento do projeto é realizado em um template do MS-Word, no qual apenas as informações que costumam diferir entre os projetos são preenchidas. Todas as informações e conhecimento que costumam se repetir nos projetos sem alteração foram definidos em documentos organizacionais que são citados nos planos específicos dos projetos. Além do template do plano do projeto, um template do cronograma no MS-Project foi elaborado contendo todas as atividades do processo padrão de desenvolvimento com a sequência e a dependência entre elas, os papéis responsáveis pela execução de cada atividade e as atividades consideradas marcos do projeto. Desta forma, o coordenador do projeto só precisa refletir as alterações realizadas na adaptação do processo padrão para o projeto e estimar o esforço e as datas de início e fim para cada atividade. Após a definição do cronograma do projeto no Project, um plugin do Redmine é utilizado para gerar uma tarefa correspondente para cada atividade do cronograma, já importando as datas de início e fim e o esforço estimado para cada atividade, que passa a ser gerenciado no Redmine. Por último, os riscos são analisados no Redmine, que permite a utilização de uma base de riscos organizacional, auxiliando na identificação dos riscos do projeto.

Para a monitoração do projeto é utilizado apenas o Redmine. A equipe do projeto reporta as datas de início e fim reais através das mudanças realizadas do estado das tarefas no Redmine e o coordenador do projeto e a equipe do projeto recebem os e-mails 
de mudança de estado das tarefas, evidenciando o andamento do projeto. Além disso, a ferramenta possui funcionalidades específicas para projetos com práticas de metodologias ágeis, como o Dashboard e o Burndown Chart do projeto. Todas as informações de andamento do projeto se encontram apenas no Redmine, o que facilita o controle do projeto por parte dos coordenadores e permite que a geração das atas de monitoração seja feita rapidamente. Nas atas constam as informações do andamento do projeto retiradas de relatórios do Redmine, os impedimentos identificados na reunião e as ações definidas para tratá-los. As ações são cadastradas e acompanhadas no Redmine.

\subsection{Gerência de Requisitos}

Não foi identificado nenhum desafio com relação a este processo. A empresa utiliza um laudo no MS-Word para avaliar os requisitos, o Enterprise Architect [Sparxsystems 2011] para a análise e projeto dos casos de uso e para a elaboração e manutenção da rastreabilidade entre os componentes do projeto, e o Redmine para o controle dos pedidos de alteração de requisitos.

\subsection{Gerência de Configuração}

O principal desafio com relação a este processo foi decidir em quais momentos seriam geradas as baselines. Ao final da Fase 1 é gerada uma baseline de planejamento. Como os sprints (Fase 2) possuem normalmente a duração de duas semanas, apesar de a literatura citar os benefícios de baselines em etapas do desenvolvimento (como especificação e análise de requisitos, codificação e testes) [Softex 2009a], optou-se por gerar apenas uma baseline ao final de cada sprint, o que não compromete a qualidade final do produto, já que as auditorias ocorrem antes de a documentação ser entregue ao cliente. Além do problema do curto período dos sprints, dentro de um mesmo sprint, cada caso de uso é tratado por vez, indo desde a sua análise até sua homologação interna, o que faz com que os documentos sejam finalizados apenas no final do sprint. Concluiu-se que, neste caso específico, baselines intermediárias travariam muito o processo, prejudicando sua agilidade e impedindo que sua duração permanecesse em duas semanas.

A ferramenta SVN [Apache 2011] é utilizada para o versionamento e controle de configuração dos documentos e código fonte. Os mecanismos de branch e tags são utilizados para gerar baselines, efetuar correções e controlar as alterações nos itens de configuração, juntamente com o Redmine, onde os pedidos de alteração são registrados e analisados. O Redmine possui integração com o SVN, possibilitando a ativação de uma restrição que exige que cada operação de commit no repositório do projeto esteja associada a alguma tarefa definida no Redmine. Os relatórios de baseline são gerados a partir da visão do repositório SVN presente no Redmine, utilizada também nas auditorias de baselines. O mesmo procedimento ocorre no escopo organizacional, que possui um projeto anual específico no Redmine.

\subsection{Garantia da Qualidade}

O principal desafio com relação a este processo foi a duração das avaliações, tanto no escopo dos projetos - onde o tempo é mais crítico - como no escopo organizacional. Inicialmente, as avaliações eram realizadas à distância por um consultor externo à empresa, o que fazia com que elas durassem cerca de dois a quatro dias entre o preenchimento inicial do laudo com a identificação das não conformidades, a compreensão e correção das não conformidades e a reavaliação das não conformidades. Decidiu-se, 
então, realizar as avaliações presencialmente, em dias e horários reservados, o que diminuiu a duração das avaliações para um a dois dias, pois no mesmo dia em que o laudo é preenchido, as não conformidades são explicadas e normalmente são corrigidas, permitindo a reavaliação logo em seguida e diminuindo o número de reavaliações por conta de correções inadequadas. Os laudos são preenchidos em templates no Excel e as não conformidades são registradas e acompanhadas no Redmine. Mensalmente, a partir da situação das não conformidades identificadas na execução dos processos, é elaborado um relatório de situação dos processos, que é apresentado para a alta direção juntamente com os resultados das análises das métricas.

\subsection{Gerência de Portfólio de Projetos}

O principal desafio com relação a este processo foi elaborar os critérios e a pontuação necessários para selecionar e priorizar os projetos para o portfólio de projetos. Os critérios estabelecidos estão relacionados a negócios, benefícios financeiros, mercado e marketing e tecnologia. Também foram criados critérios em caso de empate de pontuação. A técnica utilizada foi baseada em "weighted ranking" do The Standard for Portfolio Management [PMI 2011]. Outro desafio foi discutir estes critérios para priorização do Portfólio de Projetos com a área comercial da empresa para que a empresa tivesse a mesma cultura em todos os departamentos. Atualmente, a empresa utiliza uma planilha no Excel para a execução das atividades do processo; mais tarde, pretende-se adequar a ferramenta de apoio utilizada na área comercial para toda a execução do processo.

O Portfólio de Projeto é monitorado semanalmente ou sempre que surge uma oportunidade de negócio. Esta monitoração avalia se a carteira de projetos está atendendo aos objetivos da empresa e se cada projeto continua alinhado aos objetivos estratégicos pretendidos quando da sua aprovação. Mensalmente, é elaborado um relatório com o andamento do portfólio de projetos da empresa, que avaliado pela qualidade (evitando seu acesso aos dados sigilosos da empresa) e enviado por e-mail para os coordenadores dos projetos. A aderência ao processo é avaliada pela qualidade através de uma entrevista, na qual a execução das atividades do processo é verificada, novamente evitando seu acesso aos dados sigilosos da empresa.

\subsection{Medição}

O principal desafio com relação a este processo foi a automatização da coleta das métricas, agilizando a elaboração do relatório de medição e viabilizando reuniões mensais para apresentar suas análises, permitindo que os coordenadores dos projetos possam fazer uso das análises das medidas na gerência dos projetos. A utilização do Redmine para gerenciar todas as atividades do projeto e a definição de tipos específicos de tarefas (como planos de ação, alteração de requisitos, entre outros) permite a coleta automatizada de todas as medidas definidas pela empresa (com a exceção do formulário de satisfação do cliente ao final do projeto). Mensalmente, são utilizados alguns scripts na base de dados do Redmine que exportam os dados dos projetos para uma planilha Excel, onde gráficos são gerados para os indicadores definidos no plano de medição organizacional. Uma base OLAP foi construída para armazenar as medições com a ferramenta de Business Intelligence Pentaho [Pentaho 2011] e um processo de carga foi desenvolvido para trazer os dados do Redmine para a base de medição, permitindo que investigações possam ser realizadas nas medidas coletadas para identificar causas de problemas e possíveis soluções. 


\section{Considerações Finais}

Este trabalho apresentou a metodologia utilizada para implantar as melhorias de processos necessárias para alcançar o nível F do modelo MR-MPS na Rightway Consultoria \& Sistemas. As fases do processo padrão de desenvolvimento com as práticas da metodologia Scrum adotadas pela empresa foram explicadas. Os desafios da implementação de cada processo em um ambiente ágil foram detalhados.

A principal lição aprendida pela empresa foi que, sem um apoio ferramental adequado, a agilidade encontrada em metodologias ágeis como o Scrum pode ser prejudicada ao se implementar melhorias de processos aderentes a modelos de qualidade como o MR-MPS. A utilização do Redmine como principal ferramenta de gestão dos projetos (incluindo o projeto no escopo organizacional), juntamente com a abordagem de minimizar a repetição de informações comuns em cada projeto, permitiu que algumas atividades fossem realizadas rapidamente ou de forma automatizada. Mais detalhes sobre o apoio ferramental utilizado pela empresa podem ser encontrados em [Catunda et al. 2010]. Além disso, a alta direção e a gerência da Fábrica da empresa percebem claramente as vantagens alcançadas com as melhorias realizadas e buscam adaptar as ferramentas e processos aos objetivos da empresa, evidenciando seu apoio. Os autores esperam que estas informações possam auxiliar outras empresas e consultorias na combinação de práticas ágeis e modelos de maturidade e capacidade em seus processos de desenvolvimento.

\section{Referências}

Apache Software Foundation (2011) "Subversion (SVN)". In: http://subversion.apache.org/, accessed in 20/02/2011

Catunda, E., Nascimento, C., Cerdeiral, C.T., et al. (2010) "Implementando o nível F do MR-MPS com práticas da metodologia ágil Scrum". In: VI Workshop Anual do MPS, v. 1, pp. 78-87, Campinas, São Paulo, 26 a 29 de outubro de 2010.

Pentaho (2011). In: http://www.pentaho.com/, accessed in 20/02/2011.

PMI "Project Management Institute". In: http://www.pmi.org/, accessed in 2011.

Redmine (2011). In: http://www.redmine.org/, accessed in 20/02/2011.

Santos, G., Montoni, M., Figueiredo, S., et al. (2007a) "SPI-KM Lessons Learned from Appling a Software Process Improvement Strategy Supported by Knowledge Management". In: 8th International PROFES (Product Focused Software Development and Process Improvement), LNCS 4589, pp. 81-95, Riga, Latvia, July.

Santos, G., Montoni, M., Vasconcellos, J., et al. (2007b) "Implementing Software Process Improvement Initiatives in Small and Medium-Size Enterprises in Brazil". In: 6th International Conference on the Quality of Information and Communications Technology (QUATIC 2007)., pp. 187-198, Lisboa, Portugal, September 12-14.

ScrumAlliance (2011). In: http://www.scrumalliance.org/, accessed in 20/02/2011.

Softex (2009a) "MPS.BR - Melhoria de Processo do Software Brasileiro, Guia de Implementação - Parte 2". In: http://www.softex.br/mpsbr, accessed in 20/02/2011.

Softex (2009b) "MPS.BR - Melhoria de Processo do Software Brasileiro, Guia Geral". In: http://www.softex.br/mpsbr, accessed in 20/02/2011.

Sparxsystems (2011) "Enterprise Architect". In: http://www.sparxsystems.com.au/, accessed in 20/02/2011. 